\title{
PROSPECTS FOR IMMUNOLOGICAL CONTROL OF PARASITIC DISEASE
}

\author{
Warrant Officer 2 P. G. S. FENNELL, F.I.M.L.S., R.A.M.C. \\ The David Bruce Laboratories, East Everleigh
}

IN the majority of protozoal diseases of man, the immune response appears to be fighting a losing battle. More often than not there are specific circulating antibodies in the host and yet no effective immunity. To overcome this predicament the scientist must investigate the reasons why parasites are able to evade the hosts potentially damaging armoury of humoral and cellular immune mechanisms. The relative roles of these immune reactions have not been clearly established though it is generally assumed that humoral antibody attacks blood parasites and cell-mediated immunity attacks parasites in tissues, though of course there is great overlap.

The task facing workers in this field is not an easy one, as each parasite has its own complicated life cycle and the body's defences have to be primed ready to attack the parasite when it is most susceptible.

A natural resistance to parasites keeps at bay some 8,000 species of protozoa which are parasitic to various hosts, only about 20 protozoa being significantly pathogenic to man. These include the following:-

Rhizopoda (Entamoebae histolytica, Naegleria species, Giardia lamblia. Zoomastigophorea (Trypanosomes, Leishmania). Sporozoa (Plasmodia, Babesia, Toxoplasma, Sarcocystis) and Ciliophora (Balantidium coli). The Metazoa add a few more to the list, the Nematoda, Cestoda and Trematoda. Resistance to the other species is probably due to innate immunity though in some cases it could be due to very rapidly acquired immunity (Smithers and Terry 1969).

Specific antibody has been demonstrated to virtually all the parasites listed above and this is made use of in diagnostic intradermal and serological tests. Therefore, as a general rule, parasites effectively sensitise the host and yet continue to survive, sometimes for very long periods. The way in which parasites are able to evade these potentially lethal consequences is a matter of fundamental biological interest. Basically there are three types of clinical response to a parasite and these are:-

a. Impaired immune response. The absence of effective immunity to the parasite. This occurs in African trypanosomiasis, amoebic meningo-encephalitis, Chagas' disease, Visceral leishmaniasis, visceral larva migrans and hookworm disease.

b. Non-sterilising immunity. Persistence of parasites in the host at relatively low levels. Examples are trichinella, schistosoma and malaria. The name 'premunition' was originally given to these parasites that persist in the face of clinical immunity.

c. Sterilising immunity. Terminal evacuation of the parasite is achieved, resulting in complete resistance. This occurs in human Cutaneous leishmaniasis and in various animal protozoa.

Impaired immune response and non-sterilising immunity require greater study. Parasitic diseases are prevalent amongst so many people that any solution would be welcome. Malaria is rife amongst 500 million of the worlds population, schistosomiasis 
amongst 300 million, trypanosomiasis amongst 100 million and in many more cattle, over large areas of Africa. Helminth diseases alone affect 1000 million people and filariasis 250 million of the worlds population (Humphrey 1974).

Chemotherapy of Protozoal disease requires drugs of a selective toxicity as their biochemical pathways are similar to man and they also become resistant quite quickly, thus leaving only a narrow margin of security in treatment (Newton 1974).

It is therefore quite evident that a solution to these diseases is greatly desired and an alternative approach along immunological lines could well supply some answers. In considering the prospects for the immunological control of Parasitic diseases, it is a pre-requisite to first examine the difficulties in this field and to study the various evasion mechanisms used by the parasite.

Evasion mechanisms in the parasite are many and varied due to multiplicity of infecting protozoa, their life cycles and their divergent forms. The main evasion mechanisms are grouped under the following half-dozen titles.

a. Antigen variation. b. Immunosuppression. c. Host antigens. d. Soluble antigens. e. Survival within macrophages. f. Other evasion mechanisms.

\section{Antigen variation}

Occurring in malaria, trypanosomes, babesia and maybe also amoebae. As levels of specific antibody are formed, protozoa somehow produce new antigenic determinants, which the antibody won't recognise. The phenomenon has been studied in paramecium (Beale 1974) and possible mechanisms suggested are mutational change, recombination of diverse genetic elements, symbionts, episomes, plasmids or some similar phenomenon in protozoa, or most likely of all, switches in gene activity; variation in antigen is induced either by antibody, small temperature changes, or other physical and chemical alterations to the environment. In plasmodia it would seem to be an inductive event and not mutational.

Antigenic variation is best known to occur in trypanosomes where up to 20 variations have been shown. Trypanosomes apparently change their surface antigens by secreting a coat composed of a different glycoprotein (Vickerman 1974). Microscopy of their ultrastructure has shown an elaborate surface secretory apparatus, consisting of well developed golgi apparatus with membrane bound vesicles and tubules (Deraadt 1974). How the antigenic determinants are modified however, remains unexplained. Two antigens are thought to be present in African trypanosomes, the surface or 'variant antigens', stimulating agglutinating, neutralising and precipitating $\operatorname{IgM}$ antibodies, which are specific for each parasitaemic population, and the 'common antigens' of nuclear and cytoplasmic components which remain qualitatively unchanged during the infection. These elicit IgG antibodies which are released at the end of each parasitaemic wave, and also when trypanosome treatment commences. Most of the trypanosomes are killed by the antibodies produced, only some changing their antigenic coat. It is probably the blood forms which are taken by surprise whereas the trypanosomes in the tissues are relatively protected from neutralising antibodies.

An alternative theory suggests that antibody combines with the surface antigens, followed by capping and subsequent removal, thus exposing another layer of new 
antigens. Here capping means the gathering of similar antigens to one area of the cell membrane.

\section{Immunosuppression}

Occurring in malaria, Cutaneous leishmaniasis, trypanosomiasis and others. The parasites involved somehow suppress their host's immunological response by poorly understood mechanisms. Possible means of immunosuppression include the parasites provoking suppressor $\mathrm{T}$ cell production or non-specific factors which block effector mechanisms. Depression may be due to antigen competition, although it is doubtful how this could effect $T$ independent antibodies. Specific immunosupressive secretions by helminths is another possibility. Malaria has been shown to cause immunodepression of humoral responses to unrelated antigens such as sheep erythrocytes (Wedderburn 1974). When 51 Nigerian children with Plasmodium falciparum infections were examined, they showed diminished antibody response to tetanus and salmonella typhi vaccines, although their cell-mediated responses were normal (Greenwood 1974). Perhaps the malarial parasite has some effect on the macrophage processing of antigen. It is interesting to note that when New Zealand mice with auto-immune disease are given malaria, the mice produce lower levels of auto-antibodies. Another hypothesis suggests that due to a poly-clonal switch-on of $B$ cells, antibody production against later antigenic variants is produced and exhausted before those variants actually occur (Hudson 1974). Evidence for this is found in Trypanosoma bruce infections of mice where there is a marked increase in IgM antibodies plus an increased background response.

\section{Uptake of host antigens}

Occurring in the schistosoma and possibly the Cestoda and Trypanosoma. The adult worms of the genus schistosoma have been shown to adopt an antigenic disguise of the hosts antigens, thus masking their true identity, and enabling them to live in the mesenteric vessels of the host in comparative safety (Soulsby 1974). If schistosomal worms are taken from mice and transferred to monkeys the majority of the worms are accepted. However, if the monkey is first sensitised to mouse antigens then transferred worms are rejected. This experiment proves that either the worms are producing changed antigens to mimic the host or that the worms have acquired host antigens. It is now thought that the hosts A, B and $\mathbf{H}$ glyco-lipid antigens are absorbed by the parasite. In effect therefore, the developing schistosomula which form from the penetrating cercariae are open to immunological attack, but are soon covered with the hosts glycolipid antigens. The adult paired worms, the male in the female's gynaecophoric canal, thus secrete their worm antigens into the blood stream while they lie immune to the body's defences. The host produces anti-schistosomal antibodies to the secreted antigens, and though unable to deal with the masked invaders already present, are effective in dealing with new infections of schistosomala which will not be covered with host antigens. This differential immunity is analgous to the tumour situation and is known as ' concomitant immunity'.

Do cestodes in man have a similar mechanism for evading the immune response? Tapeworms invariably inhabit the intestines or associated organs where they stimulate little or no immunity (Ogilvie and Jones 1973). The outer surface of the proglottids are not protected by a thick barrier but by a thin membrane, through which nutrients are absorbed. Hymenolepsis microstoma in the bile duct of mice survives indefinitely, though challenge infections are severely stunted (Soulsby 1974). 
of Parasitic Disease

\section{Soluble antigens}

These occur in leishmania, trypanosomes, plasmodia, babesia, also in trichinella, nippostrongylus and schistosoma. Here the parasite evades the immune response by secreting soluble antigens which can have several different mechanisms. It can produce antigenic competition by acting on macrophages which can then inhibit phagocytosis or block cooperation between T and B cells. Soluble antigen can also produce immune complexes which could lead to $\mathrm{T}$ and $\mathrm{B}$ cell tolerance or stimulation of suppressor $T$ cells. Soluble antigen can also mop up lethal antibodies by binding and neutralising them. In many cases the secretion of soluble antigen can lead to pathological lesions in the host, as occurs in sleeping sickness when each parasitaemic wave produces a temporary excess of antigen which causes immune hypersensitivity reactions. Human erythrocytes infected with Plasmodium falciparum also release soluble antigen in vitro when the schizonts rupture; one group of these antigens (' $S$ ' antigen) is found regularly in malarial serum and could be a smokescreen blocking the immune response (Wilson 1974).

\section{Survival within macrophages}

Occurs in toxoplasmosis, leishmanial and trypanosomal infections. To survive within the body's scavenger cells is perhaps the most remarkable example of immune evasion. The parasite is protected from antibody, complement, polymorphs, lymphokines and killer cells and is able to resist the macrophage lysosomal system. For lysis to occur, lysosomes must fuse with phagosomes containing the organism and this is sometimes defective in the above infections. The host parasite combination is also important as leishmania is readily destroyed in activated macrophages of insusceptible hosts (Mauel et al 1974). Trypanosomes readily penetrate macrophages early in infections but not in the recovery stages, so therefore macrophages become activated in Trypanosoma cruzi infections (Cohen and Terry 1974).

\section{Other evasion mechanisms}

The formation of cysts by the parasite, may help to protect them as occurs with Entamoeba histolytica. Cysts formed by the host around the parasite can also be protective to both parties as in trichenella and toxoplasma infections. Hydatid cysts which are formed by both host and parasite also form an evasion mechanism. Nodules can be protective to the parasite as in Onchocerca volvulus infections where immune serum is not absorbed by living worms, but is by dead microfilaria. Refuced immunogenicity may be responsible for some nematodes developing in immune animals by undergoing adaptation as demonstrated in rats with nippostrongylus. Reduced complement $\mathrm{C}_{3}$ levels also favour increased parasitaemia in Trypanosoma cruzi infections. Immunologically privileged sites may account for the protection of some parasites as in Trichinella spirallis in muscle tissue, or microfilarial worms which reside afferent to the popliteal lymph node in mice. Trypanosomes also may occupy partially privileged sites. Outer surface being non-antigenic could account for immune evasion and it is possible that antigens responsible for the survival of many parasites are deeply embedded in the body of the organism and well protected from attack. Antigenic mimicry is another possibility whereby through evolution, parasites and their hosts share certain antigens together. 
Non-stimulation of polymorphs is seen in toxoplasma infections where the parasite seems to "turn away" the phagocytic polymorph. Quite often there is no disease caused by the parasite and both host and potential parasite live in harmony perhaps in symbiosis.

The future of immunoprophylaxis of protozoa is uncertain though there are many avenues yet to be explored. Progress in protozoal vaccines is slow and there are presently only a few animal vaccines available. A helminth vaccine of dictycaulus species is used to control sheep and cattle lungworms, and a vaccine to control dog hookworm caused by Ancylostoma caninum is also available. Both are prepared by irradiation of the infective larvae, so that they undergo some development in the host, but do not reach sexual maturity. In both instances protective immunity is by immune response to living metabolising organisms, which can be considered to be better than injection of dead parasites. Other general measures not discussed yet include that of the self-cure phenomenon which has been known to occur, for example in cases of amoebic liver, indicating that some form of resistance is at hand. Also, developing larvae of some nematodes such as ascaris in pigs and man, or toxocara in dogs, release soluble antigens which act as an anhylactic trigger releasing amines which sometimes causes expulsion of the worms. The involvement of $\mathrm{IgE}$ antibodies is in some doubt though they are invariably raised in all helminthic infections. As the macrophage is so often the final effector cell, more detailed study here is necessary. Passively transferred immunity has been tried and is successful in those diseases which would normally induce a sterilising immune response anyway.

To summarise this review I shall discuss the latest ideas and advances in the immunotherapy of protozoa, starting first with the malarial parasite. In hyperendemic areas children suffer from repeated attacks of malaria after which they remain solidly immune, so much so that it is possible to transfer passive immunity to young children terminating their malarial infections. Natural immunity to malaria appears to be genetically based, for instance Europeans in Africa are very susceptible to Plasmodium falciparium in contrast to the resistance of most Africans. The West African is also resistant to Plasmodium vivax whereas the Bantu tribes are susceptible. It is also well known that people with $\mathrm{Hb} . \mathrm{S}$, the sickle cell trait, are resistant to Plasmodium falciparium infections. The nutritional state of the host appears to be important, in that undernourished populations suffer less, and a milk diet that deprives the malarial parasite of para-aminobenzoic acid, results in low infectivity.

The key event in anti-malarial immunity would appear to be the antibodymediated inhibition of merozoite penetration into the susceptible erythrocyte. At present there are several promising approaches to vaccination and these are being extensively investigated. Studies at Guy's Hospital suggest protective immunity is related to the merozoite stage and they have had some success with this in monkeys, but the in vitro culture of merozoites is a problem. American trials of culturing Plasmodium falciparium in bone marrow cells, and in the blood of Aotus monkeys may lead to clinical trials in man soon (Leading Article 1975). The use of adjuvants is also being explored; soluble material from Plasmodium knowlesi in Freund's complete adjuvant delayed the onset of infection in the Rhesus monkey. Sufficient cross-strain immunity is necessary to transcend variation within strains and therefore perhaps the sporozoite is the best basic antigen to use. Clyde (1975) has recently tried a vaccine on three human volunteers who were exposed 
to multiple bites from an anopheles mosquito which had irradiated sporozoites of Plasmodium falciparum. One volunteer developed high immunity lasting several months. A difficulty here is obtaining sufficient sporozoites for large scale immunisation. Erythrocytic forms of the parasite from the vertebrate host are also being tested. Corradetti (1974) has successfully protected rodents against a challenge of viable Plasmodium bergei with irradiated blood stages, water insoluble fractions of this, and irradiated sporozoites. He suggests however that field trials of similar vaccines should take place in man, using human strains of plasmodia. The future of malarial vaccines therefore looks bright with many workers on the brink of a solution.

The situation is not so clear with regards to the Zoomastigophorea, the mechanics of antigenic variation requiring elucidation first. It is possible that each new variant requires the synthesis of a new enzyme and therefore an inhibitor to this is required. Trypanosoma cruzi exposed to chaotropic perchloride ions have been successfully employed as have several other vaccines for South American trypanosomiasis. Disintegrated trypanosomes offer good protection to mice for up to seven weeks. A living vaccine, attenuated with actinomycin $\mathrm{D}$ leaves the trypanosomes mobile but unable to multiply and this has also been used with some success in mice. Vaccination against African trypanosomiasis (Trypanosoma rhodesiense and Tripanosoma gambiense) seems generally less hopeful although not completely ruled out.

The survival of parasites within the macrophage is another area of study which has not yet been fully explored. Toxoplasma coated with antibody allows fusion with lysosomes and subsequent lysis, therefore, collaboration of antibodies with macrophages is necessary. More work is needed on the possibility of contact lysis of parasites, possibly by killer cells. The recruitment of lymphokines to affected areas may help in arming macrophages in an immunologically specific manner. Promotion of lysosomal fusion might also be enhanced by sub-lethal damage to the parasite.

The expulsion phase induced in nippostrongylus infections of the rat is two-fold: first antibody makes survival difficult for the parasites and then cell mediated immunity helps finally to expel the worms (Ogilvie, Personal communication 1975). Some helminths secrete enzymes to aid penetration of the host and if sufficient levels of antibody could be raised against these antigenic enzyme secretions it may be possible to prevent the worms further entry. If these soluble antigens are presented to the host in a favourable way there might well be some potential here.

Schistosomal antigens require presentation to the host without being covered with the hosts antigens. Some workers advocate the use of irradiated cercariae or deliberate infections with schistosomal species of other animals to vaccinate man. A strong and lasting immunity is induced by vaccination with irradiated hookworm larvae but it seems that the proposed agents of schistosoma vaccination would probably stimulate a much weaker and more fleeting response. Passive protection is possible in Schistosoma mansoni infections of mice and rats, and in the Rhesus monkey if transfer factor is used. Transfer factor is a leucocyte extract from an immunised animal.

Viewing the situation the most interesting question is why parasites are so often unaffected by immunity. No doubt further study into their evasion mechanisms will help to combat the parasites in their elusiveness. Future prospects in immunopropholaxis look encouraging and the next decade should show major advances in this area. 


\section{REFERENCES}

Beale, G. H. (1974). Ciba Symp. "Parasites in the Immunised_Host. Mechanisms of Survival " P.21. COHEN and Terry, R. J. (1974). Progr. Immunol. 11, 373.

Clyde, D. F. (1975). Brit. med. J. i, 231.

Corradetrt, A. (1974). Bull. Wld Hlth Org. 50, No. 3.

DeraAdT, P. (1974). Ciba Symp. "Parasites in the Immunised Host. Mechanisms of Survival" P.199.

Greenwood (1974). Ciba Symp. " Parasites in the Immunised Host. Mechanisms of Survival " P. 137-159.

Hudson, K. (1974). Progr. Immunol. 11, 375.

HuMPhrey, J. (1974). New Scientist 63, 316.

Leading Article (1975). Brit. med. J. i, 231.

MAuel, J. et al (1974). Ciba Symp. "Parasites in the Immunised Host. Mechanisms of Survival " P.225.

Newton, B. A. (1974). Ciba Symp. “Trypanosomiasis and Leishmaniasis" P.285.

OGILVIE, B. M. (1975). (Personal communication).

OGILvie, B. M. and Jones, V. E. (1973). Progr. Immunol. 17, 111.

Smithers, S. R. and Terry, R. J. (1969). Advanc. Parasit. 7, 42.

SoulsBy, E. J. L. (1974). Progr. Immunol. 11, 376.

SoulsBy, E. J. L. (1974). Progr. Immunol. 11, 377.

Vickerman, K. (1974). Ciba Symp. " Parasites in the Immunised Host. Mechanisms of Survival " P.53. Wedderburn, N. (1974). Ciba Symp. "Parasites in the Immunised Host. Mechanisms of Survival" P.123.

Wilson, R. J. M. (1974). Ciba Symp. " Parasites in the Immunised Host. Mechanisms of Survival "P.186. 